\title{
Lithium-ion batteries modeling and state of charge estimation using artificial neural network
}

\author{
Younes Boujoudar, Hassan Elmoussaoui, Tijani Lamhamdi \\ Department of Electrical Engineering, Faculty of Sciences and Technologies Fez, LSSC, \\ Sidi Mohamed Ben Abdellah University, Morocco
}

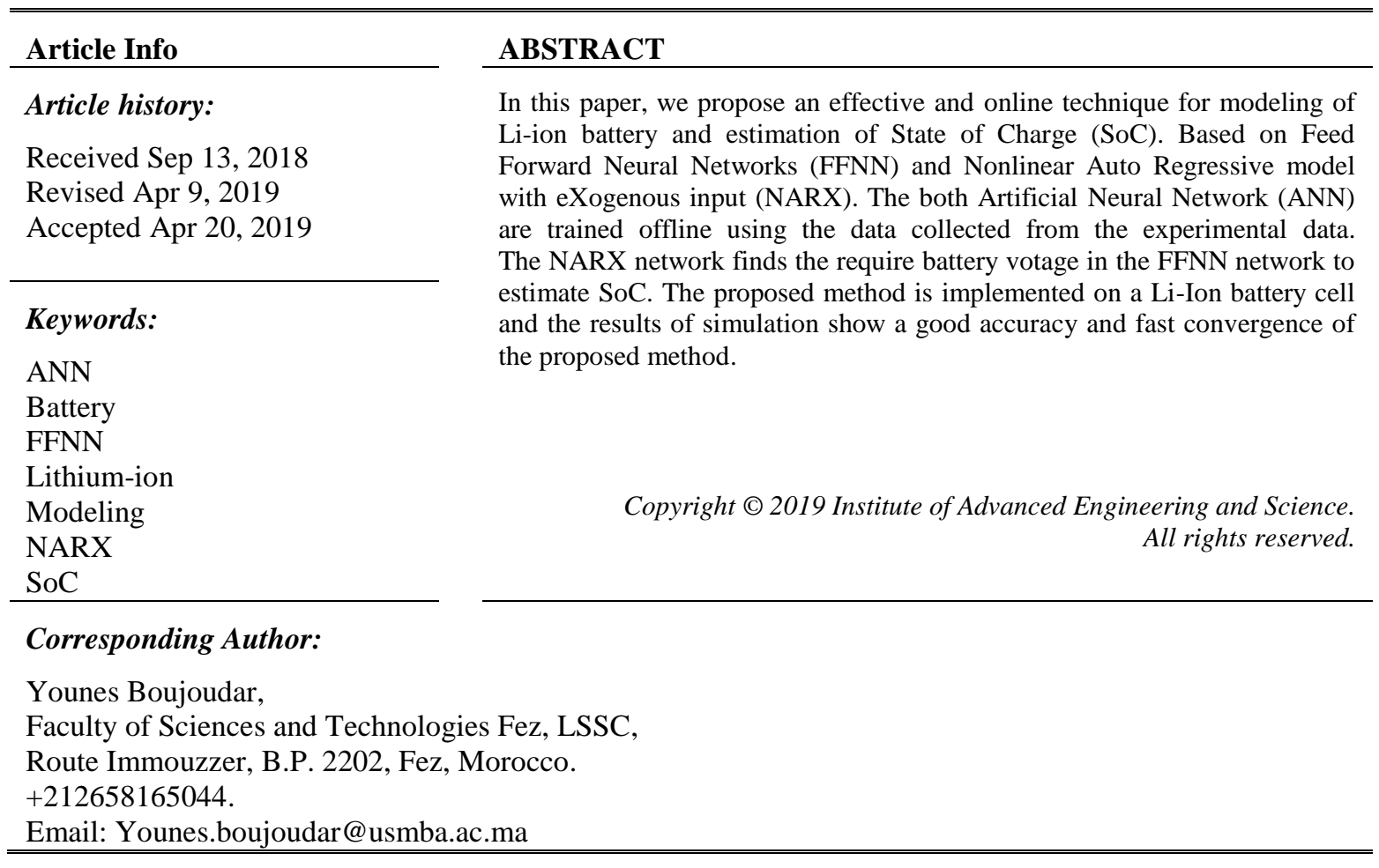

\section{INTRODUCTION}

The Lithium-Ion Batteries (LiBs) have pulled in consideration in the previous years due to their high energy density, long life cycle, and no memory effect [1]. They are used in several applications such as renewable energy systems to capture surplus electricity, electrical and hybrid vehicles, and telephone communication [2-4]. However, LiBs require exceptional caution to avoid disintegration of battery performance and prevent situations that could result in severe damage or explosions. Due to the numerous Lithium-ion batteries issues, a Battery Management Systems (BMS) is always used to guarantee safe operation [5]. The fundamental challenge of BMS is the knowledge of SoC, which represents the remaining capacity in the battery cell as a percentage of the maximum capacity. However, this state is not directly measured by any sensors [6].

For this reason, several methods to estimate SoC was proposed in the literature, depending on the choices of battery models. An accurate model representing the characteristics of the battery is essential to SoC estimation accuracy. The most used models can be summarized as two categories: the electrochemical models [7-10] and the equivalent circuit models [11-23]. The electrochemical models of battery describe the behavior of batteries based on chemical processes that happen between the electrodes and the electrolyte, there are not desirable for actual BMS [24]. Electrical circuit modeling is another useful model presented by many researchers. The simple model composed of constant internal resistance $R_{\text {in }}$ in series with a fixed ideal open circuit voltage $V_{o c}[11]$. The transient behavior of the battery has not been investigated. Therefore this model has been modified in $[12,13]$. Thevenin battery model is composed of an ideal voltage source $E_{0}$, an internal resistance $R$, overvoltage resistance $R_{0}$ and a capacitor $C_{0}$ [14]. 
A review of various Thevenin-type battery models is presented in $[15,16]$. The major disadvantage of the Thevenin model is that all parameter values are assumed to be constant [14]. References [17, 18] present a Thevenin model improvement by integration of the effects of high frequency switching and selfdischarge of the battery. Combined electrical circuit-base model proposed in [19] is consists of the voltage source (Voc(VSOC)) in series with internal resistance Rseries and $R C$ network (RtandCt), where $C t$ is the battery capacity, Ibat is the battery current, $V$ bat is the battery voltage, and Rself-discharge represents the battery discharge. This model offers circuit and system designers the possibility to improve system efficiency and prolong battery runtime for portable electronics by predicting but this model is very complicated [25]. Generic-based model based on a Shepherd equation presented in [20, 21], composed of a controlled voltage source in series with a fixed resistance. The Generic-based model is developed in [22, 23] by adding the effect of temperature and life cycle to the open circuit voltage.

In recent years, researchers on the battery model have become very popular. Due to the necessity of battery models in battery management systems and in the state of charge estimation, which represent a big problem in BMS. To solve this problem, many researchers have proposed various methods to estimate SoC: The first types of methods based on direct measurement. The most common technique for calculating the SOC is the Coulomb counting methods, also known as ampere hour counting and current integration [26, 27]. This method utilizes battery current readings mathematically integrated over time to calculate SOC values. The initial state of charge represent the problems of this methods because is unknown. Therefore this method is efficient if the initial state of charge is known. Open Circuit Voltage (OCV) method is selected to determine SoC via the relationship between OCV and SoC [28, 29].

This method is specially used for battery lead acid. Another method is discussed in [30, 31] based on the calculates of battery parameters via electrochemical spectroscopies impedance, once the parameters of the model are known, the SoC can be estimated, nevertheless this methods it is not suitable for online applications. The second type is based on battery state space mathematical models or electrical circuit battery models to design an observer for real-time SoC estimation. In [32] a Kalman Filter (KF) was proposed for estimate SoC of a lead-acid battery using the linear model. Then an Extended Kalman Filter (EKF) was studied in [33], basing on nonlinear battery models. Indeed this filter performs an analytical linearizing which causes numerical problems in the Jacobian calculate of the model [34].

The Jacobian computational problem is solved by Unscented Kalman Filter (UKF) because the Jacobian in UKF is calculate using a static linearizing. In the reference [35], a UKF is used to estimate the SoC of a Lithium-Ion battery based on a non-linear electrochemical battery model. Later an adaptive unscented Kalman filter was developed for online SoC evaluation of a Lithium-Ion battery [36]. The major drawback of this approach is that a KF needs a suitable model for battery, however, using the feedbacks in this model will require a proper state initializing for the convergence of the model.

The third category of techniques is based on the black-box battery models [37-41]. It provides the best SoC estimates due to the effective ability of computational intelligence to approximate non-linear function. Several authors [37, 38] have proposed new methods depending on Artificial Neural Network (ANN) approach. As reported in [42], a new Neural Network (NN) model was developed to estimate leadacid battery SoC, based on current measurements of discharge and temperature. Another model was presented exploiting the radial basis of the neural network function for estimating the SoC of a lead acid battery and detect the degraded pile [37]. In [39] also suggested a feed-forward neural network to estimate SoC of Ni-MH batteries. Other Artificial intelligent based methods have been investigated to compute the SoC of batteries like Fuzzy logic [40], Support Vector Machine [41].

Recently, hybrid methods were developed to improve the estimation accuracy. a hybrid method for SoC estimation based on ANN and UKF was proposed by Wei, He in Reference [43]. The state of charge SoC is determined according to the current, voltage, and temperature measured by ANN. The unscented Kalman filter is used to reduce ANN errors. Then, Radial basic neural network was used with EKF for the SoC estimation in [44]. This combined model delivers the best performance in estimating accuracy which error being less than $1 \%$ but the EKF performs an analytical linearizing which causes numerical problems in the Jacobian calculate of the model. Therefore, in this paper, a battery model of the battery is considered as a black box using a NARX model (Nonlinear Auto Regressive model with eXogenous input). Then, we used in the second feed-forward neural network to estimate SoC.

The proposed model is designed and tested on a Lithium-Ion battery. The simulation results show good accuracy and quick convergence for estimating the SoC of Li-Ion batteries. This paper is organized as follows. Section II describes the theory of neural network. Section III present the experiment data used in this paper and ANN design.Section IV details a proposed battery model and SoC estimation methods. Section IV presents the simulation results of the proposed model, and section V draws some conclusions and gives directions for the future work. 


\section{THEORY OF NEURAL NETWORK}

The Artificial Neural Network is a system motivated by the functioning of biological neurons. It is a processing architecture based on the human brain focusing on information representation by its ability to learn and adapt. They are applied in particular to solve problems of classification, prediction, categorization, and optimization. ANNs are constituted by a mathematical model of biological neuron called perceptron arranged in nodes and connected byweighing vectors or simply called weights. ANNs can model any actual data variations by constantly changing the weights between the nodes based on information flow through the network during the learning phase. ANN is well suited for modeling complex relationships between inputs and outputs with an ability to learn and adapt, therefore is at the same time a very powerful tool to model nonlinear statistical data. The basic mathematical model of ANNs is shown in Figure 1.

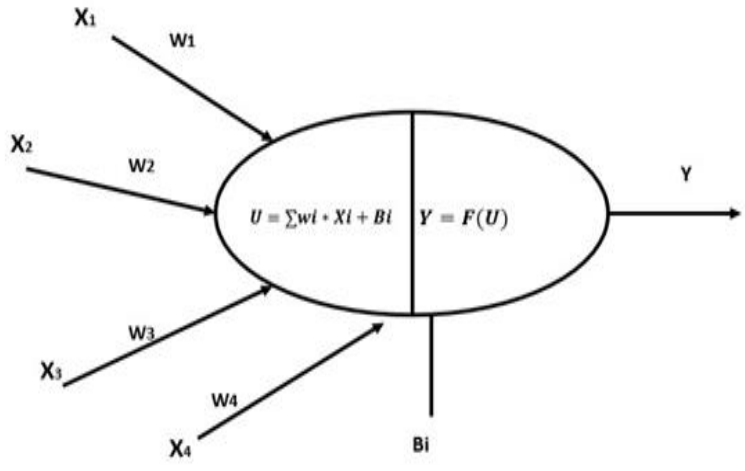

Figure 1. Neurone process

The mathematical equation of this neuron can be expressed as in (1):

$$
Y=F\left(\sum(X i * W i+B i)\right)
$$

where $X_{i}$ is the input of this neuron, $W_{i}$ is the weight of the interconnection between input $X_{i}$ and neuron, and $\mathrm{Bi}$ is the bias of this neuron. All the weights and bias are determined after the training phase.

\section{EXPERIMENT DATA AND ANN DESIGN}

Li-ion batteries are run through two different operational profiles (charge, discharge) at ambient temperatures $44 o C$. The charging was carried out in a Constant Current / Constant Voltage $(\mathrm{CC} / \mathrm{CV})$ mode. When the battery is empty the charging started by constant current at $1.5 \mathrm{~A}$ until the battery voltage arrives at $4,2 \mathrm{~V}$, and the charging continued in a constant voltage (CV) mode until the current dropped to $20 \mathrm{~mA}$. The load current is fixed at $4 \mathrm{~A}$, and the discharge voltage runs were stopped at $2.7 \mathrm{~V}$. All experiment database used in this paper is downloaded from the NASA prognostic center of excellence website [45]. The equipment required for this test is Battery Health Monitoring (BHM), sensors to measure battery voltage, current and temperature, load bank, chargers, data acquisition system and a computer for control and analysis.

The NN training can be made more efficient and robust through proper normalization of the data [43]. Therefore, before training, the inputs were normalized to the range [-1; 1$]$ by:

$$
X N=\frac{2(X-X \min )}{X \max -X \min }-1
$$

where Xmin and Xmax are the minimum and maximum in the input vector X of the NN. In the testing step, the testing data was scaled using the same Xmin and Xmax used in the training data.

After building the database passed to the separation of the learning and validation basis. Generally, there is no precise rule concerning this separation but in a general way the validation database represents from $10 \%$ to $25 \%$ of the general database. Once the two databases are created, it will be necessary to define an architecture of the neural network. We use an FFNN and NARX models trained with the backpropagation learning algorithm, to its capacity to solve nonlinear problem. The risk of over-learning has always existed when we used an artificial neural network. Therefore the optimization is a primary phase in 
the design of the neural network. The objective of optimization step is to locate the optimal design of the neural network, we performed several tests to find the optimal numbers of hidden layer and numbers of neuron per layer.

The performances of the ANN will be measured by Mean Squared Error (MSE). The results of neural network performance in the optimization process are presented in Table 1. The optimal architecture is with 10 neurons in the first and the second hidden layer.

Table 1. Neural network optimisation results

\begin{tabular}{ccc}
\hline Neural network architecture & PERFORMANCE(MSE) & Max error at validation \\
\hline$[5,5]$ & $1.564 \mathrm{e}-01$ & 0.56 \\
{$[8,8]$} & $6.954 \mathrm{e}-03$ & 0.23 \\
{$[10,10]$} & $2.2249 \mathrm{e}-04$ & 0.10 \\
{$[12,12]$} & $2.212 \mathrm{e}-04$ & 0.12 \\
{$[12,14]$} & $2.18749 \mathrm{e}-04$ & 0.132 \\
\hline
\end{tabular}

\section{PROPOSED BATTERY MODEL}

Li-ion battery is a complicated system to modeling due to the nonlinearity of voltage response. ANNs, are found to be good universal approximates which approximate any function to desired accuracy. The proposed model is designed with two neural networks, the first based on NARX model to find the battery voltage at the sampling time $\mathrm{k}$ as a function of the voltage, SoC at the sampling time $k-1$, and the current, temperature at the sampling time k. as it is presented in the Figure 2. The second to estimate SoC is based on FFNN (Feed-Forward Neural Network). The structure of FFNN is shown in Figure 3, where the inputs are the battery voltage at sampling time $k-1$ and the measurement of current and temperature at sampling time $k$.

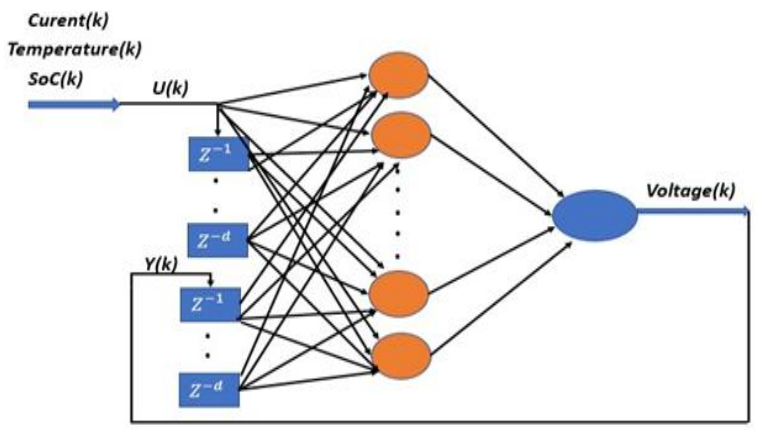

Figure 2. NARX Model

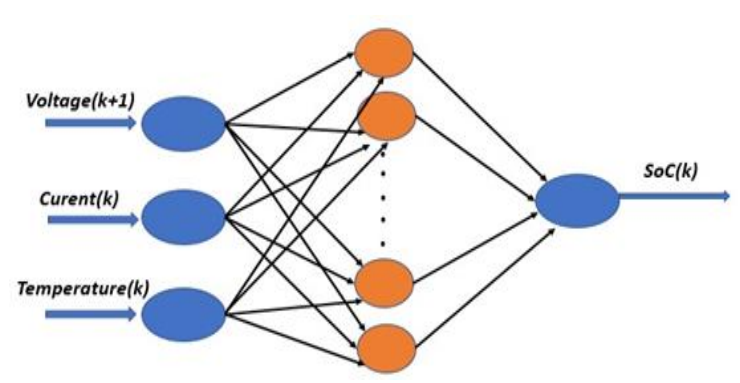

Figure 3. FFNN for SoC estimation

From the both models of Fig. 2 and 3 we can obtained the relationship of the output $Y(k)$ as a function of Input $U(k)$ and previous output $Y(k-1), Y(k-2)$ for the NARX model:

$$
Y(k)=F(U(k), U(k-1) \ldots, U(k-d), Y(k-1), \ldots Y(k-d))
$$

The function $F$ is the hyperbolic tangent, often used in the hidden layer as an activation function and linear transfer function in the output layer.

$$
F(u)=\frac{2}{1+\exp (-2 * u)}-1
$$

All parameters of the two neural networks are obtained after the step of neural network training using backpropagation algorithms. The battery is modeled using a NARX model, which is trained using the data obtained from the battery, and the state of charge is estimated using FFNN. The global proposed system is show in Figure 4. 


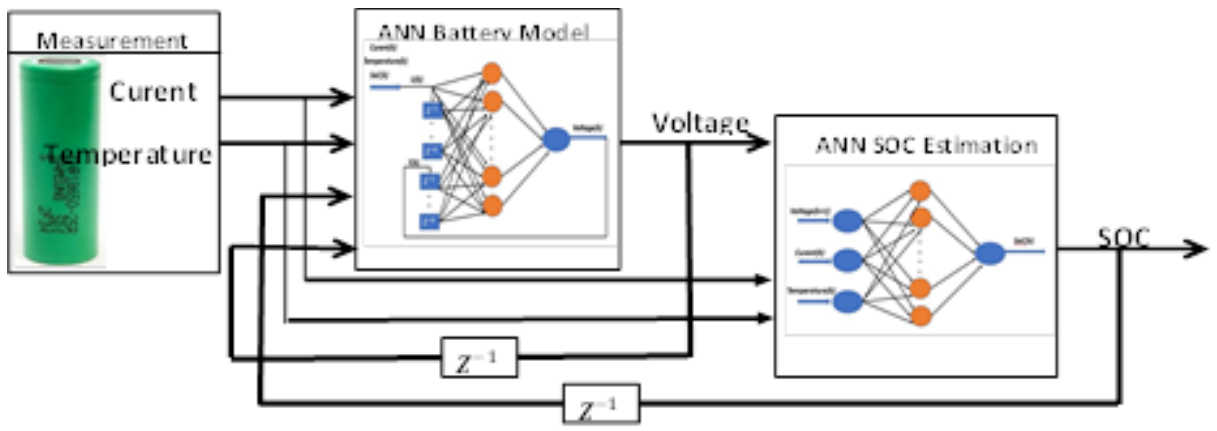

Figure 4. Structure of proposed battery model

Since the SoC of the battery is one of the inputs to the NN, it is necessary to measure the SoC using one of the available methods. For this reason, the ampere-hour counting technique, given in [26, 27], is employed for collecting the training data.

\section{RESULTS AND DISCUSSION}

This paper proposes a new battery model and SoC estimation. The originality of our work lies in the fact that battery model is dynamic, take into account the effect of temperature and SoC on the battery models, and we are used this model to estimate SoC. The battery was charged from $0 \%$ to $100 \%$ and discharged from $100 \%$ to $0 \%$, so the integration error was negligible because the current sensor was well calibrated, therefore the solid curve is regarded as the experimental SoC [43]. We can see in the next figures four results of SoC and voltage shows the complete similarity between the reference and the estimated voltage and SoC during the charge and discharge process for a learning and validation data. The Figure 5 shows the comparison between the experiment SoC and the output of FFNN for training data, and the Figure 6 for the validation data. The Figure 7 present the output of NARX model and experiment battery voltage for training data, and the Figure 8 for the validation data.

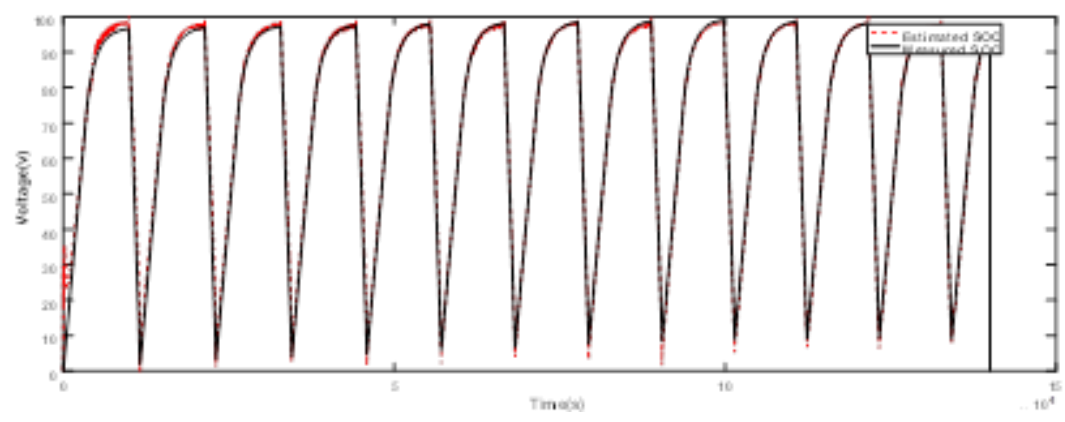

Figure 5. Experimental and estimated battery SoC (training data)

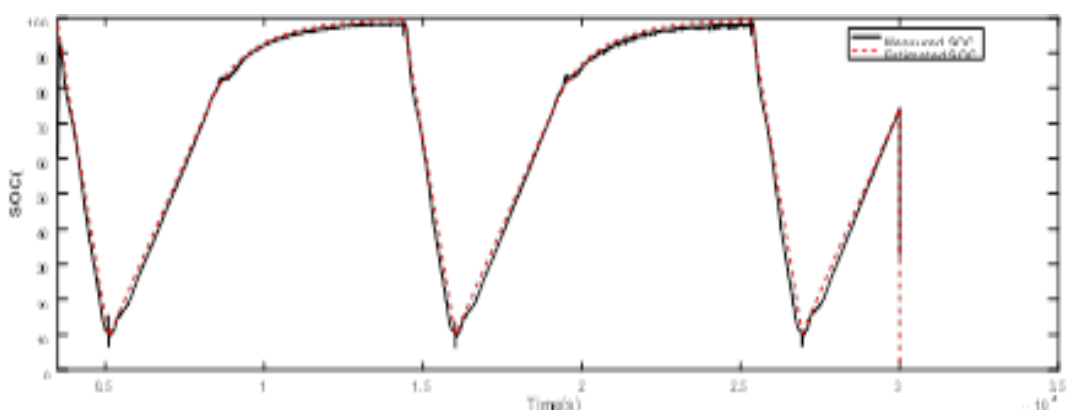

Figure 6. Experimental and estimated battery SOC (validation data) 


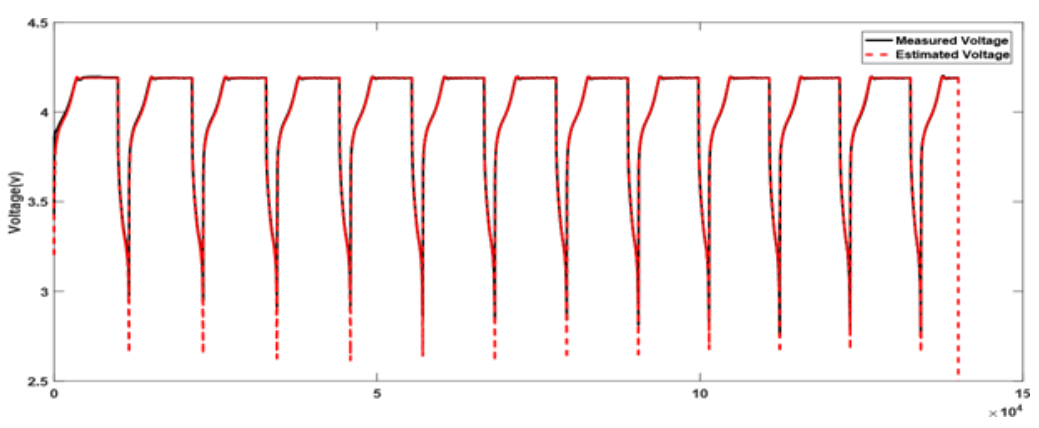

Figure 7. Experimental and estimated battery voltage (training data)

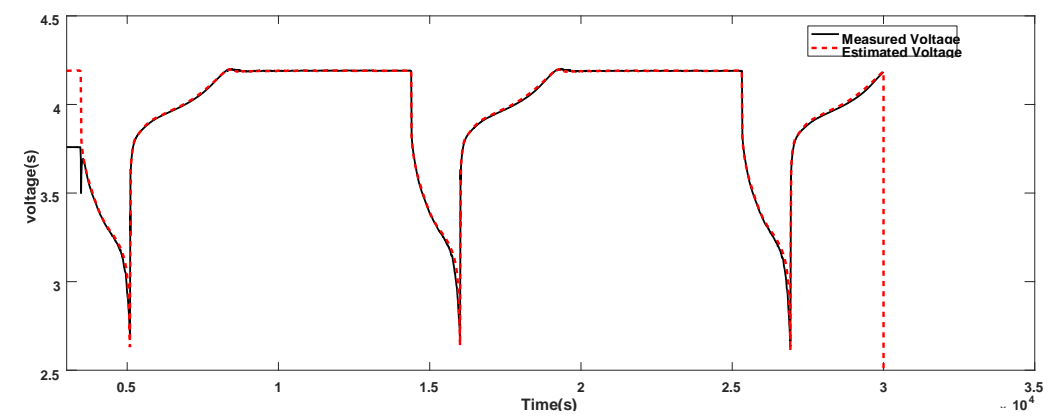

Figure 8. Experimental and estimated battery voltage (validation data)

The maximum error of NARX model is $4 \%$ but we can see some error peak after each discharge of battery due to the high degree of battery discharge. This peak shows the robustness of our model because the curve of our model converges quickly to the experimental curve. For the second model FFNN the maximum error is $10 \%$, due to the peak error of battery voltage after each discharge operation, and due to error propagation in both neural network.

\section{CONCLUSION}

A SoC estimator system for Li-Ion batteries using ANN was proposed in this paper. The ANN is of NARX and FFNN types. The NARX was trained off-line to find the appropriate model needed in the FFNN, which estimates the SoC of the battery. All experiment database used in this paper is downloaded from the NASA prognostic center of excellence web site. The simulation results of the proposed estimator showed good accuracy and fast convergence to the experimental variable, independent of the charging conditions. The proposed model could be used for several rechargeable batteries. Therefore some challenges about the proposed model need to discuss here. For applying this model to a battery pack, it is necessary to calculate SoC for each cell. The batteries are used in the different environmental condition. Therefore the database used in the design of this model needs to contain all the possible operation scenario. For the next work.

\section{REFERENCES}

[1] H. R. Eichi, et al., "Battery management system: An overview of its application in the smart grid and electric vehicles," IEEE Industrial Electronics Magazine, vol/issue: 7(2), pp. 4-16, 2013.

[2] Sutopo, et al., "Designing Framework for Standardization Case Study: Lithium-Ion Battery Module in Electric Vehicle Application,” International Journal of Electrical and Computer Engineering, vol/issue: 8(1), pp. 220, 2018.

[3] S. K. Bhuyan, et al., "Modeling, Control and Power Management Strategy of a Grid connected Hybrid Energy System," International Journal of Electrical and Computer Engineering, vol/issue: 8(3), pp. 1345, 2018.

[4] J. M. Azeroual, et al., "Renewable energy potential and available capacity for wind and solar power in morocco towards 2030," Journal of Engineering Science and Technology Review, vol/issue: 11(1), pp. 189-198, 2018.

[5] C. Alaoui, "Thermal management for energy storage system for smart grid," Journal of Energy Storage, vol. 13, pp. 313-324, 2017. 
[6] S. Tong, et al., "Battery state of charge estimation using a load-classifying neural network," Journal of Energy Storage, vol. 7, pp. 236-243, 2016.

[7] T. F. Fuller, et al., "Simulation and optimization of the dual lithium ion insertion cell," Journal of the Electrochemical Society, vol/issue: 141(1), pp. 1-10, 1994.

[8] C. Wang and V. Srinivasan, "Computational battery dynamics (cbd) electrochemical/thermal coupled modeling and multi-scale modeling," Journal of power sources, vol/issue: 110(2), pp. 364-376, 2002.

[9] V. R. Subramanian, et al., "Mathematical model reformulation for lithium-ion battery simulations: Galvanostatic boundary conditions," Journal of The Electrochemical Society, vol/issue: 156(4), pp. A260-A271, 2009.

[10] X. Zhang, et al., "Electrochemical-electrical-thermal modeling of a pouch-type lithium ion battery: An application to optimize temperature distribution," Journal of Energy Storage, vol. 11, pp. 249-257, 2017.

[11] V. Johnson, "Battery performance models in advisor," Journal of power sources, vol/issue: 110(2), pp. 321-329, 2002.

[12] M. Durr, et al., "Dynamic model of a lead acid battery for use in a domestic fuel cell" system," Journal of power sources, vol/issue: 161(2), pp. 1400-1411, 2006.

[13] H. Chan, "A new battery model for use with battery energy storage systems and electric vehicles power systems," in Power Engineering Society Winter Meeting, 2000. IEEE, Vol. 1, IEEE, pp. 470-475, 2000.

[14] Z. M. Salameh, et al., "A mathematical model for lead-acid batteries," IEEE Transactions on Energy Conversion, vol/issue: 7(1), pp. 93-98, 1992.

[15] M. Nikdel, et al., "Various battery models for various simulation studies and applications," Renewable and Sustainable Energy Reviews, vol. 32, pp. 477-485, 2014.

[16] H. He, et al., "Comparison study on the battery models used for the energy management of batteries in electric vehicles," Energy Conversion and Management, vol. 64, pp. 113-121, 2012.

[17] J. Appelbaum and R. Weiss, "An electrical model of the lead-acid battery," in Telecommunications Energy Conference, 1982. INTELEC 1982. International, IEEE, pp. 304-307, 1982.

[18] F. M. G. Longatt, "Circuit based battery models: A review," in Congreso Iberoamericano de estudiantes' De Ingenieria Electrica. Cibelec, 2006.

[19] M. Chen and G. A. R. Mora, "Accurate electrical battery model capable of predicting runtime and iv performance," IEEE transactions on energy conversion, vol/issue: 21(2), pp. 504-511, 2006.

[20] O. Tremblay, et al., "A generic battery model for the dynamic simulation of hybrid electric vehicles," in Vehicle Power and Propulsion Conference, 2007. VPPC 2007. IEEE, pp. 284-289, 2007.

[21] O. Tremblay and L. A. Dessaint, "Experimental validation of a battery dynamic model for ev applications," World Electric Vehicle Journal, vol/issue: 3(1), pp. 1-10, 2009.

[22] H. Hemi, et al., "Dynamic modeling and simulation of temperature and current effects on an electric vehicles lithium ion battery," in Electrical and Computer Engineering (CCECE), 2015 IEEE 28th Canadian Conference on, IEEE, pp. 970-975, 2015.

[23] Y. Boujoudar, et al., "Li-ion battery parameters estimation using neural networks," in Wireless Technologies, Embedded and Intelligent Systems (WITS), 2017 International Conference on, IEEE, pp. 1-4, 2017.

[24] K. A. Smith, et al., "Model-based electrochemical estimation and constraint management for pulse operation of lithium ion batteries," IEEE Transactions on Control Systems Technology, vol/issue: 18(3), pp. 654-663, 2010.

[25] A. Khalil, et al., "The impact of the time delay on the load frequency control system in microgrid with plug-inelectric vehicles," Sustainable Cities and Society, vol. 35, pp. 365-377, 2017.

[26] K. S. Ng, et al., "Enhanced coulomb counting method for estimating state-of-charge and state-of-health of lithiumion batteries," Applied energy, vol/issue: 86(9), pp. 1506-1511, 2009.

[27] S. Jeon, et al., "Comparative study on the battery state-of-charge estimation method," Indian Journal of Science and Technology, vol/issue: 8(26), 2015.

[28] Y. Xing, et al., "State of charge estimation of lithium-ion batteries using the open-circuit voltage at various ambient temperatures," Applied Energy, vol. 113, pp. 106-115, 2014.

[29] K. S. Ng, et al., "State-of-charge estimation for lead-acid batteries based on dynamic open-circuit voltage," in Power and Energy Conference, 2008. PECon 2008. IEEE 2nd International, IEEE, pp. 972-976, 2008.

[30] A. Guha, et al., "Remaining useful life estimation of lithium-ion batteries based on the internal resistance growth model," in Control Conference (ICC), 2017 Indian, IEEE, pp. 33-38, 2017.

[31] J. P. R. Barrera, et al., "Soc estimation for lithium-ion batteries: Review and future challenges," Electronics, vol/issue: 6(4), pp. 102, 2017.

[32] B. S. Bhangu, et al., "Nonlinear observers for predicting state-of-charge and state-of-health of lead-acid batteries for hybrid-electric vehicles," IEEE transactions on vehicular technology, vol/issue: 54(3), pp. 783-794, 2005.

[33] G. L. Plett, "Extended kalman filtering for battery management systems of lipb-based hev battery packs: Part 3. state and parameter estimation," Journal of Power sources, vol/issue: 134(2), pp. 277-292, 2004.

[34] A. Murangira, "Nouvelles approches en filtrage particulaire. application au recalage de la navigation inertielle," Ph.D. thesis, Universite de Technologie de Troyes-UTT, 2014.

[35] S. Santhanagopalan and R. E. White, "State of charge estimation using an unscented filter for high power lithium ion cells," International Journal of Energy Research, vol/issue: 34(2), pp. 152-163, 2010.

[36] F. Sun, et al., "Adaptive unscented kalman filtering for state of charge estimation of a lithium-ion battery for electric vehicles," Energy, vol/issue: 36(5), pp. 3531-3540, 2011

[37] W. Shen, et al., "A new battery available capacity indicator for electric vehicles using neural network," Energy Conversion and Management, vol/issue: 43(6), pp. 817-826, 2002. 
[38] Y. Morita, et al., "On-line detection of state-of-charge in lead acid battery using radial basis function neural network," Asian Journal of Control, vol/issue: 8(3), pp. 268-273, 2006.

[39] B. Cheng, et al., "Ni-mh batteries state-of-charge prediction based on immune evolutionary network," Energy Conversion and Management, vol/issue: 50(12), pp. 3078-3086, 2009.

[40] A. J. Salkind, et al., "Determination of state-of-charge and state-of-health of batteries by fuzzy logic methodology," Journal of Power sources, vol/issue: 80(1-2), pp. 293-300, 1999.

[41] J. A. Ant'on, et al., "Battery state-of-charge' estimator using the svm technique," Applied Mathematical Modelling, vol/issue: 37(9), pp. 6244-6253, 2013.

[42] O. V. S. R. Kumar and K. Srikanth, "Energy losses and global emissions reduction with distributed generation and case study on impact of temperature conditions within a micro grid."

[43] W. He, et al., "State of charge estimation for li-ion batteries using neural network modeling and unscented kalman filter-based error cancellation," International Journal of Electrical Power \& Energy Systems, vol. 62, pp. 783-791, 2014.

[44] M. Charkhgard and M. Farrokhi, "State-of-charge estimation for lithium-ion batteries using neural networks and ekf," IEEE transactions on industrial electronics, vol/issue: 57(12), pp. 4178-4187, 2010.

[45] B. Saha and K. Goebel, "Battery data set," NASA AMES prognostics data repository, 2007. 